\title{
Parity index of binary words and powers of prime words
}

\author{
Aleksandar Ilić \\ Faculty of Sciences and Mathematics \\ University of Niš, Serbia \\ aleksandari@gmail.com
}

\author{
Sandi Klavžar \\ Faculty of Mathematics and Physics \\ University of Ljubljana, Slovenia \\ and \\ Faculty of Natural Sciences and Mathematics \\ University of Maribor, Slovenia \\ sandi.klavzar@fmf .uni-lj.si
}

Submitted: Apr 1, 2012; Accepted: Sep 15, 2012; Published: Oct 4, 2012

Mathematics Subject Classifications: 05A05, 68R15, 68W32.

\begin{abstract}
Let $f$ be a binary word and let $\mathcal{F}_{d}(f)$ be the set of words of length $d$ which do not contain $f$ as a factor (alias words that avoid the pattern $f$ ). A word is called even/odd if it contains an even/odd number of 1s. The parity index of $f$ (of dimension $d$ ) is introduced as the difference between the number of even words and the number of odd words in $\mathcal{F}_{d}(f)$. A word $f$ is called prime if every nontrivial suffix of $f$ is different from the prefix of $f$ of the same length. It is proved that if $f$ is a power of a prime word, then the absolute value of the parity index of $f$ is at most 1 . We conjecture that no other word has this property and prove the conjecture for words $0^{r} 1^{s} 0^{t}, r, s, t \geqslant 1$. The conjecture has also been verified by computer for all words $f$ of length at most 10 and all $d \leqslant 31$.
\end{abstract}

Keywords: binary words; combinatorics on words; words avoiding a pattern; parity index; generalized Fibonacci cubes.

${ }^{*}$ Corresponding author 


\section{Introduction}

Elements of $B=\{0,1\}$ are called bits and an element of $B^{d}$ is a binary word of length $d$. Since all words considered here are binary, we will simply speak about words. A word $u \in B^{d}$ will be written in the coordinate form as $u=u_{1} u_{2} \ldots u_{d}$. A word $f$ is a factor of a word $x$ if $f$ appears as a sequence of $|f|$ consecutive bits of $x$. A word $u$ is called $f$-free if it does not contain $f$ as a factor. For a word $f$ and positive integer $d$, let

$$
\mathcal{F}_{d}(f)=\left\{u \in B^{d} \mid u \text { is } f \text {-free }\right\} .
$$

The product notation will mean concatenation, for example, $1^{r}$ is the word of length $r$ with all bits equal 1. A word $b$ is a power of a word $c$ if $b=c^{k}$ for some $k \geqslant 1$. A word is called even if it contains an even number of $1 \mathrm{~s}$ and odd otherwise.

Suppose that $f$ is a word and $d$ is a positive integer. Then the generalized Fibonacci cube, $Q_{d}(f)$, is the graph obtained from the $d$-dimensional cube $Q_{d}$ by removing all vertices that contain $f$ as a factor. In other words, $V\left(Q_{d}(f)\right)=\mathcal{F}_{d}(f)$, two vertices being adjacent if they differ in exactly one bit. These graphs were studied for the first time in [4], but special cases were extensively studied earlier. The most notable special case is formed by Fibonacci cubes $\Gamma_{d}=Q_{d}(11), d \geqslant 1$, see the survey [6]. The special case of $Q_{d}\left(1^{s}\right)$ was introduced in [3] (under the same name of generalized cubes) and further investigated in $[8,12]$.

The definition of the generalized Fibonacci cubes naturally leads to different problems on words. The most fundamental problem is to determine the order of these graphs. This problem was studied earlier under the notion of words avoiding a pattern. Calling $f$ a pattern, then the number of words avoiding $f$ is just the number of $f$-free words. Baccherini, Merlini and Sprugnoli [1] were interested in the number of $f$-free words that contain prescribed numbers of $0 \mathrm{~s}$ and $1 \mathrm{~s}$ and established that they are closely related to proper Riordan arrays. This work was extended in [9].

Another natural problem about generalized Fibonacci cubes is when they embed isometrically into hypercubes. This question naturally leads to the concept of the so called good and bad words. A word $f$ is said to be $d$-good if for any $f$-free words $u$ and $v$ of length $d, v$ can be obtained from $u$ by complementing one by one the bits of $u$ on which $u$ and $v$ differ, such that all intermediate words are $f$-free. Then $f$ is good if it is $d$-good for any $d \geqslant 1$. The main result of [7] asserts that about eight percent of all words are good. The study of good words was continued in [5] by introducing the index $\beta(f)$ of $f$ as the smallest integer $d$ such that $f$ is not $d$-good. Among other results it was proved that $\beta(f)<|f|^{2}$ holds for any bad word $f$.

Our principal motivation for the present paper is a result of [8] asserting that each $Q_{d}\left(1^{r}\right)$ contains a hamiltonian path. This in particular implies that the bipartition of $Q_{d}\left(1^{r}\right)$ is balanced. (By the way, it is not difficult to see that every generalized Fibonacci cube is connected.) Clearly, the bipartition sets of $Q_{d}(f)$ are formed by even and odd words, respectively. Hence, for a set of words $X$, let $e(X)$ and $o(X)$ be the number of even and odd words in $X$, respectively. Let in addition $\Delta(X)=e(X)-o(X)$, in particular write $\Delta(x)=\Delta(\{x\})$ for a word $x$. That is, $\Delta(x)=1$ if $x$ is even and $\Delta(x)=-1$ if $x$ is 
odd. Then we define the parity index of $f$ of dimension $d$ as

$$
\mathrm{PI}_{d}(f)=\Delta\left(\mathcal{F}_{d}(f)\right) .
$$

Using this notation, a necessary condition for $Q_{d}(f)$ to contain a hamiltonian path is that $\left|\mathrm{PI}_{d}(f)\right| \leqslant 1$.

In the next section we introduce prime words and prove that if $f$ is a power of a prime word then $\left|\mathrm{PI}_{d}(f)\right| \leqslant 1$ holds for any $d$. In Section 3 we consider the parity index of the words $0^{r} 1^{s} 0^{t}$ and prove that for any $d$ large enough, $\left|\mathrm{PI}_{d}\left(0^{r} 1^{s} 0^{t}\right)\right| \geqslant 2$. For the special case of $0^{r} 10^{r}$ a more precise result is obtained, in particular it is noted that $\left\{\left|P I_{d}(010)\right|\right\}_{d \geqslant 3}$ is the so-called Padovan sequence. In Section 4 we pose a conjecture that powers of prime words are the only words with the property $\left|\mathrm{PI}_{d}(f)\right| \leqslant 1$ for any $d$ and verify the conjecture for all words of length $\leqslant 10$ and for all $d \leqslant 31$. We conclude by indicating a possible approach to the conjecture via generating functions that count the words that do not contain the binary word as a given factor.

\section{Powers of prime words}

A word $f$ of length $d$ is prime if for any $k, 1 \leqslant k \leqslant d-1$, the suffix of $f$ of length $k$ is different from the prefix of $f$ of the same length. In particular, words 0 and 1 are prime, and if $d \geqslant 2$, then the first bit and the last bit of a prime word are different. For instance, 001101 is a prime word which easily follows from the fact that the factor 00 appears only at its beginning. On the other hand the word 01101011 is not prime as it starts and ends with 011.

For a word $f$ of length $\ell$ let $\mathcal{S}_{d}(f)=B^{d} \backslash \mathcal{F}_{d}(f)$, that is,

$$
\mathcal{S}_{d}(f)=\left\{b=b_{1} b_{2} \ldots b_{d} \mid b \text { contains factor } f\right\} .
$$

For $i=1,2, \ldots, d-\ell+1$ let in addition

$$
\mathcal{S}_{d}^{(i)}(f)=\left\{b=b_{1} b_{2} \cdots b_{d} \mid b \in S, b_{i} b_{i+1} \cdots b_{i+\ell-1}=f\right\} .
$$

Then $\mathcal{S}_{d}(f)=\bigcup_{i=1}^{d-\ell+1} \mathcal{S}_{d}^{(i)}(f)$.

By $\left(\begin{array}{l}X \\ k\end{array}\right)$ we denote the set of all $k$-subsets of the set $X$.

Lemma 1. Let $f$ be a word of length $\ell$. Then

$$
\Delta\left(\mathcal{S}_{d}(f)\right)=\sum_{k=1}^{d-\ell+1}(-1)^{k-1} \sum_{I \subseteq\left(\begin{array}{c}
\mathbb{N}_{d}-\ell+1 \\
k
\end{array}\right)} \Delta\left(\cap_{i \in I} \mathcal{S}_{d}^{(i)}(f)\right) .
$$

Proof. Let $\chi_{A}$ be the characteristic function of a set $A$ :

$$
\chi_{A}(x)= \begin{cases}1 ; & x \in A \\ 0 ; & \text { otherwise } .\end{cases}
$$


Since $\mathcal{S}_{d}(f)=\bigcup_{i=1}^{d-\ell+1} \mathcal{S}_{d}^{(i)}(f)$, the inclusion and exclusion principle implies that for every $x \in \mathcal{S}_{d}(f)$,

$$
\sum_{k=1}^{d-\ell+1}(-1)^{k-1} \sum_{I \subseteq\left(\mathbb{N}_{d-\ell+1}^{k}\right)} \chi_{\cap_{i \in I} \mathcal{S}_{d}^{(i)}(f)}(x)=1
$$

Therefore,

$$
\begin{aligned}
& \Delta\left(\mathcal{S}_{d}(f)\right)=\sum_{x \in \mathcal{S}_{d}(f)} \Delta(x) \\
& =\sum_{x \in \mathcal{S}_{d}(f)} \Delta(x)\left(\sum_{k=1}^{d-\ell+1}(-1)^{k-1} \sum_{I \subseteq\left(\begin{array}{c}
\mathbb{N}_{d-\ell+1} \\
k
\end{array}\right)} \chi_{\cap_{i \in I} \mathcal{S}_{d}^{(i)}(f)}(x)\right) \\
& =\sum_{k=1}^{d-\ell+1}(-1)^{k-1} \sum_{I \subseteq\left(\begin{array}{c}
\mathbb{N}_{d-\ell+1} \\
k
\end{array}\right)} \sum_{x \in \mathcal{S}_{d}(f)} \Delta(x) \chi_{\cap_{i \in I} \mathcal{S}_{d}^{(i)}(f)}(x) \\
& =\sum_{k=1}^{d-\ell+1}(-1)^{k-1} \sum_{I \subseteq\left(\begin{array}{c}
\left.\mathbb{N}_{d-\ell+1}\right) \\
k
\end{array}\right)} \Delta\left(\cap_{i \in I} \mathcal{S}_{d}^{(i)}(f)\right) \text {. }
\end{aligned}
$$

Theorem 2. Let $f$ be a power of a prime word. Then $\left|\mathrm{PI}_{d}(f)\right| \leqslant 1$ for any $d \geqslant 1$.

Proof. Let $d \geqslant 1$. Suppose first that $f$ is a prime word. When $d<\ell$, we have $\mathcal{F}_{d}(f)=B^{d}$ and if $d=\ell$, then $\mathcal{F}_{d}(f)$ contains all but the word $f$. Hence we may assume in the rest that $d>\ell$. Since $e\left(B^{d}\right)=o\left(B^{d}\right)$, we have $e\left(\mathcal{F}_{d}(f)\right)+e\left(\mathcal{S}_{d}(f)\right)=o\left(\mathcal{F}_{d}(f)\right)+o\left(\mathcal{S}_{d}(f)\right)$. Hence $\operatorname{PI}_{d}(f)=\Delta\left(\mathcal{F}_{d}(f)\right)=-\Delta\left(\mathcal{S}_{d}(f)\right)$. It thus suffices to prove that $\left|\Delta\left(\mathcal{S}_{d}(f)\right)\right| \leqslant 1$.

We first note that $\Delta\left(\mathcal{S}_{d}^{(i)}(f)\right)=0$. Indeed, the first $i-1$ bits and the last $d-\ell-i+1$ bits of the words from $\mathcal{S}_{d}^{(i)}(f)$ are arbitrary, hence $\mathcal{S}_{d}^{(i)}(f)$ contains $2^{d-\ell-1}$ even words and the same number of odd words. Consider now $X=\cap_{i \in I} \mathcal{S}_{d}^{(i)}(f)$ where $I=\left\{i_{1}, i_{2}, \ldots, i_{k}\right\}$ and $i_{1}<i_{2}<\cdots<i_{k}$. Because $f$ is a prime word, $X=\emptyset$ as soon as for some index $j$, $i_{j+1}-i_{j}<\ell$. Moreover, by the same argument as the one used for $\Delta\left(\mathcal{S}_{d}^{(i)}(f)\right), \Delta(X)=0$ as soon as for some index $j, i_{j+1}-i_{j}>\ell$. Hence $\Delta(X)$ can be nonzero only when $k \ell=d$ and $i_{j}=(j-1) \ell+1$ for each $1 \leqslant j \leqslant k$. Therefore, applying Lemma 1 ,

$$
\Delta\left(\mathcal{S}_{d}(f)\right)=\left\{\begin{aligned}
0 ; & \ell \nmid d, \\
-1 ; & \ell \mid d, k \text { odd, } f \text { contains odd number of } 1 \mathrm{~s}, \\
1 ; & \text { otherwise. }
\end{aligned}\right.
$$

The proof is complete for a prime word $f$.

Assume now that $f=\left(f^{\prime}\right)^{r}$, where $f^{\prime}$ is a prime word and $r \geqslant 2$. Let $\left|f^{\prime}\right|=\ell^{\prime}$. The proof continues similarly as in the case when $f$ was prime. The only difference is that now $X=\emptyset$ as soon as for some index $j$, the difference $i_{j+1}-i_{j}$ is not a multiple of $\ell^{\prime}$ and so $\Delta(X)$ can be nonzero only when $d$ is a multiple of $\ell^{\prime}$. 


\section{Non-prime words}

In this section we study the parity index of words consisting of three blocks, that is, of words $0^{r} 1^{s} 0^{t}, r, s, t \geqslant 1$. Clearly, none of these words is prime. In our main result (Theorem 4) we prove that for no such word $f,\left|\mathrm{PI}_{d}(f)\right| \leqslant 1$ holds for all $d$. Before that we separately give a more precise result for the special case of $0^{r} 10^{r}$. The obtained results in particular imply that $Q_{d}\left(0^{r} 1^{s} 0^{t}\right)$ does not contain a hamiltonian path as soon as $d$ is large enough.

Theorem 3. Let $r \geqslant 1$. Then

$$
\left|\mathrm{PI}_{d}\left(0^{r} 10^{r}\right)\right|= \begin{cases}0 ; & d \leqslant 2 r, 2 r+2 \leqslant d \leqslant 3 r+1 \\ 1 ; & d=2 r+1,3 r+2 \leqslant d \leqslant 4 r+3 .\end{cases}
$$

Moreover, for any $d \geqslant 4 r+4,\left|\mathrm{PI}_{d}\left(0^{r} 10^{r}\right)\right| \geqslant 2$.

Proof. Suppose first that $d \leqslant 2 r$. Then $\mathcal{F}_{d}\left(0^{r} 10^{r}\right)=B^{d}$ and hence $\mathrm{PI}_{d}\left(0^{r} 10^{r}\right)=0$. Since $\mathcal{F}_{2 r+1}\left(0^{r} 10^{r}\right)=B^{d} \backslash\left\{0^{r} 10^{r}\right\}$ we have $\mathrm{PI}_{2 r+1}\left(0^{r} 10^{r}\right)=1$.

Let $d \geqslant 2 r+2$. Recall that $-\mathrm{PI}_{d}\left(0^{r} 10^{r}\right)=\Delta\left(\mathcal{S}_{d}(f)\right)=\sum_{b \in \mathcal{S}_{d}(f)} \Delta(b)$. By Lemma 1 ,

$$
-\mathrm{PI}_{d}(f)=\sum_{k=1}^{d-\ell+1}(-1)^{k-1} \sum_{I \subseteq\left(\begin{array}{c}
\mathbb{N}_{d-\ell+1} \\
k
\end{array}\right)} \Delta\left(\cap_{i \in I} \mathcal{S}_{d}^{(i)}(f)\right)
$$

Suppose that for a set $X=\cap_{i \in I} \mathcal{S}_{d}^{(i)}(f)$ there exists an index $i$ such that if $w \in X$ then also $w+e_{i} \in X$. Then $\Delta(X)=0$. It follows that $\Delta(X) \neq 0$ if and only if there exist $k \geqslant 0$ and $r \leqslant r_{j} \leqslant 2 r$ for all $1 \leqslant j \leqslant k$, such that

$$
X=\left\{0^{r} 10^{r_{1}} 10^{r_{2}} 1 \cdots 0^{r_{k}} 10^{r}\right\} .
$$

Moreover, in that case $\Delta(X)=\Delta\left(0^{r} 10^{r_{1}} 10^{r_{2}} 1 \cdots 0^{r_{k}} 10^{r}\right)=(-1)^{k+1}$.

Hence let $k \geqslant 0$ and $r \leqslant r_{j} \leqslant 2 r, 1 \leqslant j \leqslant k$, and set $b=0^{r} 10^{r_{1}} 10^{r_{2}} 1 \cdots 0^{r_{k}} 10^{r}$. Let $v=b_{r_{1}+2} b_{t_{1}+3} \cdots b_{d}$ be the word obtained from $b$ by omitting the first $r_{1}+1$ bits, so that $v \in B^{d-r_{1}-1}$. Since $b$ has one more bit of 1 than $v$ does, $\Delta(v)=-\Delta(b)$.

Note that $v$ starts with $0^{r} 1$. Then

$$
v \in \bigcap_{j \in J} S_{d-r_{1}-1}^{(j)}(f) \quad \text { if and only if } \quad b \in \mathcal{S}_{d}^{(1)}(f) \bigcap\left(\cap_{j \in J} \mathcal{S}_{d}^{\left(j+r_{1}+1\right)}(f)\right) .
$$

Now we can compute as follows:

$$
\begin{aligned}
\mathrm{PI}_{d}\left(0^{r} 10^{r}\right) & =-\Delta\left(\mathcal{S}_{d}(f)\right)=-\sum_{b \in \mathcal{S}_{d}(f)} \Delta(b) \\
& =-\left(\sum_{\substack{b \in \mathcal{S}_{d}(f) \\
r_{1}=r}} \Delta(b)+\sum_{\substack{b \in \mathcal{S}_{d}(f) \\
r_{1}=r+1}} \Delta(b)+\cdots+\sum_{\substack{b \in \mathcal{S}_{d}(f) \\
r_{1}=2 r}} \Delta(b)\right)
\end{aligned}
$$




$$
\begin{aligned}
&=-\left(\sum_{v \in S_{d-r-1}(f)}-\Delta_{d-r-1}(v)+\sum_{v \in S_{d-r-2}(f)}-\Delta_{d-r-2}(v)+\cdots\right. \\
&\left.+\sum_{v \in S_{d-2 r-1}(f)}-\Delta_{d-2 r-1}(v)\right) \\
&= \sum_{v \in S_{d-r-1}(f)} \Delta_{d-r-1}(v)+\sum_{v \in S_{d-r-2}(f)} \Delta_{d-r-2}(v)+\cdots \\
&+\sum_{v \in S_{d-2 r-1}(f)} \Delta_{d-2 r-1}(v) \\
&= \sum_{r \leqslant r_{1} \leqslant 2 r} \sum_{v \in S_{d-r_{1}-1}(f)} \Delta_{d-r_{1}-1}(v) \\
&= \sum_{r \leqslant r_{1} \leqslant 2 r} \Delta_{d-r_{1}-1}\left(\begin{array}{l}
v \\
=
\end{array}\right. \\
& \sum_{r \leqslant r_{1} \leqslant 2 r} \Delta_{d-r_{1}-1}\left(S_{d-r_{1}-1}(f)\right) \\
&=-\sum_{r \leqslant r_{1} \leqslant 2 r} \mathrm{PI}_{d-r_{1}-1}\left(0^{r} 10^{r}\right) .
\end{aligned}
$$

It follows that $\left|\mathrm{PI}_{d}\left(0^{r} 10^{r}\right)\right|=\left|\sum_{r \leqslant r_{1} \leqslant 2 r} \mathrm{PI}_{d-r_{1}-1}\left(0^{r} 10^{r}\right)\right|$.

As the values $\mathrm{PI}_{d-r_{1}-1}\left(0^{r} 10^{r}\right)$ have the same sign for all $r \leqslant r_{1} \leqslant 2 r$, from Equation (1) we get

$$
\left|\mathrm{PI}_{d}\left(0^{r} 10^{r}\right)\right|=\sum_{r \leqslant r_{1} \leqslant 2 r}\left|\mathrm{PI}_{d-r_{1}-1}\left(0^{r} 10^{r}\right)\right|
$$

Set $a_{d}=\left|\mathrm{PI}_{d}\left(0^{r} 10^{r}\right)\right|$ for all $d$. We already know that $a_{d}=0$ for all $d \leqslant 2 r$ and that $a_{2 r+1}=1$. Let $d \geqslant 2 r+2$. If $d \leqslant 3 r+1$ and there is a word $b \in S_{d}(f)$, then there is an index $i$ such that if $w \in X$ then also $w+e_{i} \in X$ and hence $\Delta(X)=0$.

Assume $d \geqslant 3 r+2$. When $3 r+2 \leqslant d \leqslant 4 r+2, \mathrm{PI}_{d}\left(0^{r} 10^{r}\right)=\Delta\left(0^{r} 10^{d-2 r-2} 10^{r}\right)=1$ or -1 and hence $a_{d}=1$. When $d=4 r+3, a_{d}=a_{2 r+2}+\cdots+a_{3 r+2}=1$. Let $d=4 r+3+u$ for some $u \geqslant 1$. Then by Equation (2), $a_{4 r+3+u}=a_{2 r+2+u}+\cdots+a_{3 r+2+u}$. If $u \leqslant r$, then $2 r+2+u \leqslant 3 r+2<3 r+2+u \leqslant 4 r+2$ and therefore $a_{d} \geqslant 2$. Assume $u \geqslant r+1$. Let $u^{\prime}=$ $u-r$. Then $d=5 r+3+u^{\prime}$ for $u^{\prime} \geqslant 1$. By Equation (2), $a_{5 r+3+u^{\prime}}=a_{3 r+2+u^{\prime}}+\cdots+a_{4 r+2+u^{\prime}}$. If $u^{\prime} \leqslant r$, then $3 r+2 \leqslant 3 r+2+u^{\prime}<4 r+3 \leqslant 4 r+2+u^{\prime}$ and therefore $a_{d} \geqslant 2$. Assume $u^{\prime} \geqslant r+1$. Then let $u^{\prime \prime}=u^{\prime}-r$. Then $d=6 r+3+u^{\prime \prime}$ for $u^{\prime \prime} \geqslant 1$. By Equation (2), $a_{6 r+3+u^{\prime \prime}}=a_{4 r+2+u^{\prime \prime}}+\cdots+a_{5 r+2+u^{\prime \prime}}$. As $3 r+2 \leqslant 4 r+2+u^{\prime \prime}<5 r+2+u^{\prime \prime}, a_{d} \geqslant 2$. Thus when $d \geqslant 4 r+4, a_{d} \geqslant 2$.

The special case of Theorem 3 when $r=1$ deserves a special attention. In that case,

$$
\left|\mathrm{PI}_{d}(010)\right|=\left|\mathrm{PI}_{d-2}(010)\right|+\left|\mathrm{PI}_{d-3}(010)\right|
$$


with initial conditions $\left|\mathrm{PI}_{3}(010)\right|=1,\left|\mathrm{PI}_{4}(010)\right|=0,\left|\mathrm{PI}_{5}(010)\right|=1$ which is the Padovan sequence, see sequence A000931 from [11].

Theorem 4. Let $r, s, t \geqslant 1$. Let $z$ be the integer such that $(z-1) t+2 \leqslant r+s \leqslant z t+1$. Then

$$
\left|\mathrm{PI}_{d}\left(0^{r} 1^{s} 0^{t}\right)\right|\left\{\begin{aligned}
=0 ; & d<r+s+t \\
& y(r+s+t)<d<(y+1)(r+s)+t \quad \text { for } 1 \leqslant y \leqslant z, \\
\geqslant 1 ; & d=r+s+t, \\
& (y+1)(r+s)+t \leqslant d \leqslant(y+1)(r+s+t) \quad \text { for } 1 \leqslant y \leqslant z, \\
& d=(z+1)(r+s+t)+1 .
\end{aligned}\right.
$$

Moreover, for any $d \geqslant(z+1)(r+s+t)+2,\left|\mathrm{PI}_{d}\left(0^{r} 1^{s} 0^{t}\right)\right| \geqslant 2$.

Proof. Since $\mathrm{PI}_{d}\left(0^{r} 1^{s} 0^{t}\right)=\mathrm{PI}_{d}\left(0^{t} 1^{s} 0^{r}\right)$, it suffices to prove the result for words $0^{r} 1^{s} 0^{t}$ with $r \geqslant t$. By the same argument as in the proof of Theorem $3, \Delta(X) \neq 0$ if and only if there exist $k \geqslant 0$ and $r \leqslant r_{j} \leqslant r+t$ for all $1 \leqslant j \leqslant k$ such that

$$
X=\left\{0^{r} 1^{s} 0^{r_{1}} 1^{s} 0^{r_{2}} 1^{s} \cdots 0^{r_{k}} 1^{s} 0^{t}\right\}
$$

where $\Delta(X)=(-1)^{(k+1) s}$. Also

$$
\left|\mathrm{PI}_{d}\left(0^{r} 1^{s} 0^{t}\right)\right|=\sum_{r \leqslant r_{1} \leqslant r+t}\left|\mathrm{PI}_{d-r_{1}-s}\left(0^{r} 1^{s} 0^{t}\right)\right|
$$

Set $a_{d}=\left|\mathrm{PI}_{d}\left(0^{r} 1^{s} 0^{t}\right)\right|$ for all $d$. We already know that $a_{d}=0$ for all $d<r+s+t$ and that $a_{r+s+t}=1$. Let $d \geqslant r+s+t+1$. In the first part of the proof, we prove the theorem for $d \leqslant(z+1) r+(z+1) s+(z+1) t$ by induction on $y$ for $1 \leqslant y \leqslant z$. Then we prove the theorem for $d \geqslant(z+1) r+(z+1) s+(z+1) t+1$. The idea of the proof is as follows. From the first part of the proof, we notice that for each $y \geqslant 1, a_{d}=0$ for $r+s-(y-1) t-1$ consecutive numbers of $d$ and then $a_{d} \geqslant 1$ for the next $y t+1$ consecutive numbers of $d$. As $y$ increases, $r+s-(y-1) t-1$ decreases to zero and $y t+1$ increases. While, by Equation (3), $a_{d}=a_{d-r-s-t}+\cdots+a_{d-r-s}$, which is a sum of $t+1$ consecutive numbers, where $t+1$ is a constant for given $0^{r} 1^{s} 0^{t}$. Therefore for large enough $d, a_{d} \geqslant 2$.

By a similar argument as in the proof of Theorem 3, $a_{d}=0$ if $d<2 r+2 s+t$ and $a_{d}=1$ if $2 r+2 s+t \leqslant d \leqslant 2 r+2 s+2 t$. Thus the statement is true for $y=1$. Let $y \geqslant 2$. Suppose the statement is true for all $1 \leqslant y_{0}<y$. Let $d=y r+y s+y t+u$ for some $u \geqslant 1$. Then by Equation (3), $a_{y r+y s+y t+u}=a_{(y-1) r+(y-1) s+(y-1) t+u}+\cdots+a_{(y-1) r+(y-1) s+y t+u}$. When $d<(y+1) r+(y+1) s+t$, i.e., $u<r+s-(y-1) t,(y-1) r+(y-1) s+(y-1) t<$ $(y-1) r+(y-1) s+(y-1) t+u<(y-1) r+(y-1) s+y t+u<y r+y s+t$ and hence by the induction assumption, $a_{d}=0$. When $d=(y+1) r+(y+1) s+t$, i.e., $u=r+s-(y-1) t$, $(y-1) r+(y-1) s+y t+u=y r+y s+t$ and hence by the induction assumption, $a_{d} \geqslant a_{y r+y s+t} \geqslant 1$. Assume $d \geqslant(y+1) r+(y+1) s+t+1$, i.e., $u \geqslant r+s-(y-1) t+1$. Let $u^{\prime}=u-r-s+(y-1) t$. Then $d=(y+1) r+(y+1) s+t+u^{\prime}$ where $u^{\prime} \geqslant 1$. By Equation (3), $a_{d}=a_{y r+y s+u^{\prime}}+\cdots+a_{y r+y s+t+u^{\prime}}$. If $d \leqslant(y+1) r+(y+1) s+(y+1) t$, i.e., $u^{\prime} \leqslant y t$, then $y r+y s+u^{\prime} \leqslant y r+y s+y t$. Considering that $y r+y s+t<y r+y s+$ 
$t+u^{\prime}, a_{d} \geqslant a_{y r+y s+t}+a_{y r+y s+t+1}$ or $a_{d} \geqslant a_{y r+y s+u^{\prime}}+a_{y r+y s+u^{\prime}+1}$ depending on whether $y r+y s+u^{\prime}<y r+y s+t$ or not. Therefore by the induction assumption, $a_{d} \geqslant 1$. Thus the theorem is proved for all $d \leqslant(z+1) r+(z+1) s+(z+1) t$.

Assume $d \geqslant(z+1) r+(z+1) s+(z+1) t+1 \geqslant(z+2) r+(z+2) s+t$. Let $d=$ $(z+2) r+(z+2) s+t+u^{\prime \prime}$ where $u^{\prime \prime} \geqslant 0$. Then by Equation (3), $a_{d}=a_{(z+2) r+(z+2) s+t+u^{\prime \prime}}=$ $a_{(z+1) r+(z+1) s+u^{\prime \prime}}+\cdots+a_{(z+1) r+(z+1) s+t+u^{\prime \prime}}$. Note that $(z+1) r+(z+1) s+t+u^{\prime \prime} \geqslant$ $(z+1) r+(z+1) s+t$. Assume $d=(z+2) r+(z+2) s+t$, i.e., $u^{\prime \prime}=0$. Then $(z+1) r+(z+$ 1) $s+t+u^{\prime \prime}=(z+1) r+(z+1) s+t$. If $r+s>z t$, then $(z+1) r+(z+1) s>z r+z s+z t$ and hence $a_{d}=a_{(z+1) r+(z+1) s+t} \geqslant 1$. If $r+s \leqslant z t$, then $(z+1) r+(z+1) s \leqslant z r+z s+z t$ and hence $a_{d} \geqslant a_{z r+z s+z t}+a_{(z+1) r+(z+1) s+t} \geqslant 2$.

Let $d>(z+2) r+(z+2) s+t$, i.e., $u^{\prime \prime}>0$. Then $(z+1) r+(z+1) s+t+u^{\prime \prime}>$ $(z+1) r+(z+1) s+t$. First assume $d<(z+2) r+(z+2) s+(z+2) t$, i.e., $u^{\prime \prime}<(z+1) t$. Then $(z+1) r+(z+1) s+u^{\prime \prime}<(z+1) r+(z+1) s+(z+1) t$. Therefore $a_{d} \geqslant a_{(z+1) r+(z+1) s+t}+$ $a_{(z+1) r+(z+1) s+t+1}$ or $a_{d} \geqslant a_{(z+1) r+(z+1) s+u^{\prime \prime}}+a_{(z+1) r+(z+1) s+u^{\prime \prime}+1}$ depending on whether $(z+1) r+(z+1) s+u^{\prime \prime}<(z+1) r+(z+1) s+t$ or not. Thus $a_{d} \geqslant 2$ in any case. Second assume $d=(z+2) r+(z+2) s+(z+2) t$, i.e., $u^{\prime \prime}=(z+1) t$. Then $(z+1) r+(z+1) s+u^{\prime \prime}=$ $(z+1) r+(z+1) s+(z+1) t$ and hence $a_{d} \geqslant a_{(z+1) r+(z+1) s+u^{\prime \prime}}+a_{(z+1) r+(z+1) s+u^{\prime \prime}+1} \geqslant 2$ considering that $(z+1) r+(z+1) s+(z+1) t<(z+1) r+(z+1) s+u^{\prime \prime}+1<(z+2) r+$ $(z+2) s+(z+2) t$. Finally assume $d>(z+2) r+(z+2) s+(z+2) t$, i.e., $u^{\prime \prime}>(z+1) t$. Suppose there is $u^{\prime \prime}>(z+1) t$ such that $a_{d} \leqslant 1$. Let $u_{0}^{\prime \prime}$ be the smallest such an integer and $d_{0}=(z+2) r+(z+2) s+t+u_{0}^{\prime \prime}$. Then $a_{d_{0}}=a_{(z+1) r+(z+1) s+u_{0}^{\prime \prime}}+\cdots+a_{(z+1) r+(z+1) s+t+u_{0}^{\prime \prime}}$. Since $(z+1) r+(z+1) s+u_{0}^{\prime \prime}>(z+1) r+(z+1) s+(z+1) t$ and $(z+1) r+(z+1) s+t+u_{0}^{\prime \prime}<d_{0}$, $a_{d_{0}} \geqslant 2$, which is a contradiction. Thus the statement is true for all $d$.

\section{Computer evidence and conjecture}

Using computer we obtained the parity index for all words $f$ of length at most 10 and all $d \leqslant 31$. Since $Q_{d}(f)$ is isomorphic to $Q_{d}(\bar{f})$, where $\bar{f}$ is the binary complement of $f$, we have restricted the computation to words $f$ that contain not more 1 s than 0s. From the same reason reversed words need not to be considered. In Table 1 all words $f$ of length at most 8 and with $\left|\mathrm{PI}_{d}(f)\right| \leqslant 1$ for $d \leqslant 31$ are collected.

It can be checked that every word from the table is a power of a prime word. Moreover, the same was verified also for the obtained words of length 9 and 10 (not given in the table). Based on this experiment and Theorems 2 and 4 we pose:

Conjecture 5. Let $f$ be a word such that $\left|\mathrm{PI}_{d}(f)\right| \leqslant 1$ holds for any $d$. Then $f$ is a power of a prime word.

A possible approach to the conjecture would be to prove that if $f$ is not a power of a prime word, then the sequence $\left\{\left|\mathrm{PI}_{d}(f)\right|\right\}_{d}$ satisfies a certain recurrence relation from which we can deduce the behavior of the sequence. For instance, one can establish the recurrent formula

$$
\left|\mathrm{PI}_{d}(01110)\right|=\left|\mathrm{PI}_{d-4}(01110)\right|+\left|\mathrm{PI}_{d-5}(01110)\right|
$$




\begin{tabular}{l|l}
\hline length & $f$ \\
\hline 3 & 001 \\
\hline 4 & $0001,0011,0101$ \\
\hline 5 & $00001,00011,00101$ \\
\hline 6 & $000001,000011,000101,000111$ \\
& $001001,001011,001101,010101$ \\
\hline 7 & $0000001,0000011,0000101,0000111$ \\
& $0001001,0001011,0001101,0010011$ \\
\hline 8 & 0010101,0011101 \\
\hline & $00000001,00000011,00000101,00000111$ \\
& $0001001,00001011,00001101,00001111$ \\
& $00011001,00010011,00010101,00010111$ \\
& $00100101,00101011,00011101,00100011$ \\
& $00110101,00111101,01010101,00110011$ \\
\hline
\end{tabular}

Table 1: List of words $f$ with $|f| \leqslant 8$ and $\left|\mathrm{PI}_{d}(f)\right| \leqslant 1$ for $d \leqslant 31$

with initial conditions $\left|\mathrm{PI}_{5}(01110)\right|=1,\left|\mathrm{PI}_{6}(01110)\right|=\left|\mathrm{PI}_{7}(01110)\right|=\left|\mathrm{PI}_{8}(01110)\right|=0$ and $\left|\mathrm{PI}_{9}(01110)\right|=1$. Similarly, either by applying Equation (3) or by a tedious case analysis yields, one can get:

$$
\begin{aligned}
\left|\mathrm{PI}_{d}(000001000)\right|= & \left|\mathrm{PI}_{d-6}(000001000)\right|+\left|\mathrm{PI}_{d-7}(000001000)\right| \\
& +\left|\mathrm{PI}_{d-8}(000001000)\right|+\left|\mathrm{PI}_{d-9}(000001000)\right|,
\end{aligned}
$$

with initial conditions $\left|\mathrm{PI}_{9}(000001000)\right|=1,\left|\mathrm{PI}_{10}(000001000)\right|=\left|\mathrm{PI}_{11}(000001000)\right|=$ $\left|\mathrm{PI}_{12}(000001000)\right|=\left|\mathrm{PI}_{13}(000001000)\right|=\left|\mathrm{PI}_{14}(000001000)\right|=0,\left|\mathrm{PI}_{15}(000001000)\right|=$ $\left|\mathrm{PI}_{16}(000001000)\right|=\left|\mathrm{PI}_{17}(000001000)\right|=1$.

In Fig. 1 the values of $\left|\mathrm{PI}_{d}(01110)\right|$ and $\left|\mathrm{PI}_{d}(000001000)\right|$ for $5 \leqslant d \leqslant 55$ are plotted. Note that the sequence $\left|P I_{d}(f)\right|$ does not need to be monotone, but it seems that starting from some large enough dimension the sequence is strictly increasing.

\section{Concluding remarks}

Another approach to get further insight into the parity index of a binary word (in particular into Conjecture 5) would be to investigate the relationship with the generating function counting the words that do not contain the binary word as a given factor. To explicitly state these functions we need the following concepts from [2], see also [10, page 374] and [1,9]. Let $f=f_{0} f_{1} \ldots f_{d-1}$ be a word of length $d$, then its autocorrelation $c^{[f]}=c_{0} c_{1} \ldots c_{d-1}$ is the binary word with $c_{i}$ defined to be 1 if $f_{j}=f_{i+j}$ for all 


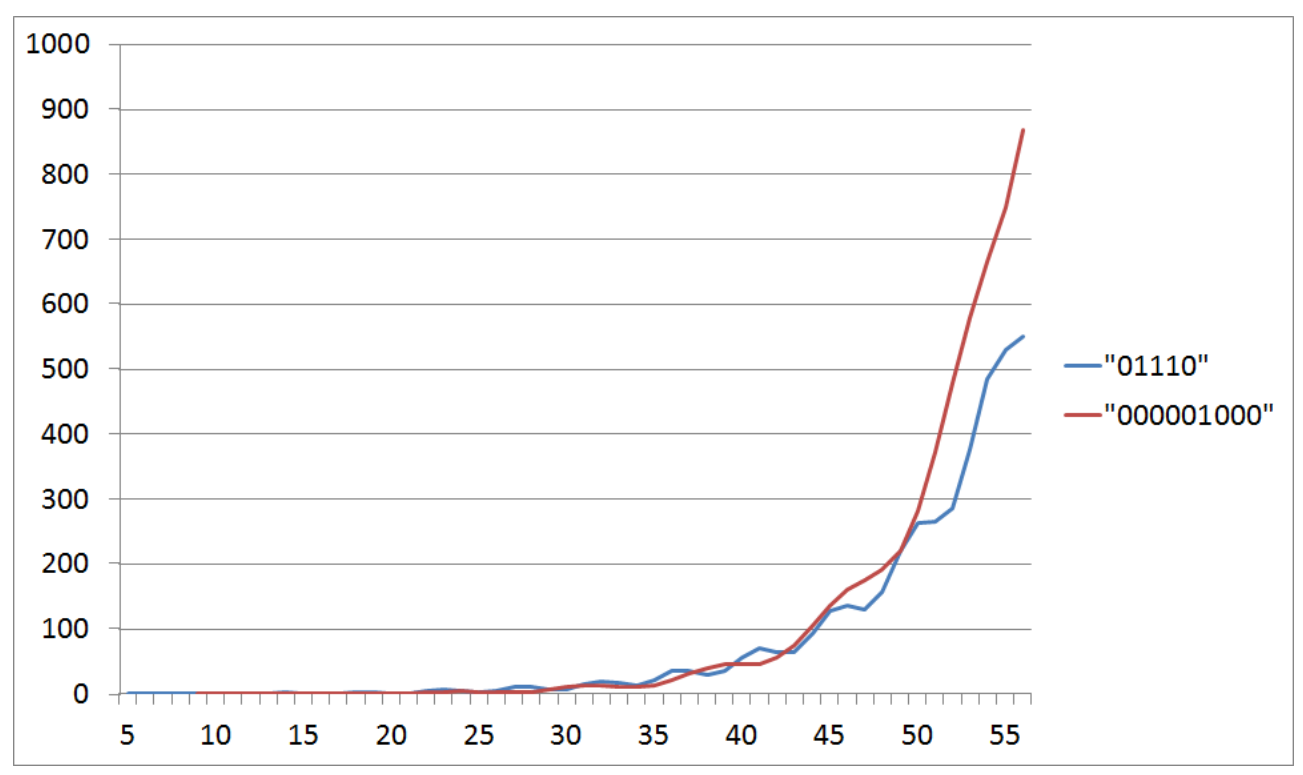

Figure 1: Values of $\left|\mathrm{PI}_{d}(f)\right|$ for $f=01110$ and $f=000001000$

$0 \leqslant j \leqslant d-1-i$, and defined to be $c_{i}=0$ otherwise. (In other words, $c_{i}=1$ if $f$ and its shift by $i$ bits agree in their intersection. In the terminology from [7] we could also say that $c_{i}=1$ when $f$ has a 0 -error overlap of length $d-i$.) For instance, the autocorrelation of 1100111 is 1000011 and the one of 101010 is 101010. The autocorrelation polynomial $C^{[f]}(x, y)$ is the polynomial with coefficients given by the autocorrelation of $f$. More precisely, the coefficient of $x^{j} y^{i}$ is 1 if $c_{j+i}^{[f]}=1$ and the suffix of $f$ (of length $i+j$ ) contains $j$ ones and $i$ zeros. For instance, $C^{[1100111]}(x, y)=1+x^{3} y^{2}+x^{4} y^{2}$. Then the generating function counting the binary words that do not contain $f$ as a factor is (see $[1,9]$ ):

$$
F^{[f]}(x, y)=\frac{C^{[f]}(x, y)}{(1-x-y) C^{[f]}(x, y)+x^{d_{1}} y^{d_{0}}},
$$

where $d_{1}$ and $d_{0}$ are the numbers of ones and zeros in $f$, respectively, and the symbols $x$ and $y$ count the number of ones and zeros in the words.

It could be interesting to notice the relation between the forbidden words studied here and the shape of the corresponding autocorrelation polynomials as well as to approach $\left|\mathrm{PI}_{d}(f)\right|$ in terms of $F^{[f]}(x, y)$. To these two ideas we also add that it could also be interesting to relate the $r$-error overlap studies from [7] with the respective autocorrelation polynomials.

\section{Acknowledgements}

We are grateful to a referee for pointing us the approach to the parity index as discussed in Section 5.

This work was supported by the research Grants 174010 and 174033 of Serbian Ministry of Education and Science, by the research grant P1-0297 of Ministry of Higher Edu- 
cation, Science and Technology Slovenia, and by Basic Science Research Program through

the National Research Foundation of Korea funded by the Ministry of Education, Science and Technology grant 2011-0025319.

\section{References}

[1] D. Baccherini, D. Merlini and R. Sprugnoli. Binary words excluding a pattern and proper Riordan arrays. Discrete Math., 307:1021-1037, 2007.

[2] L. J. Guibas and A. M. Odlyzko. String overlaps, pattern matching, and nontransitive games. J. Combin. Theory Ser. A, 30:183-208, 1981.

[3] W.-J. Hsu. Fibonacci cubes - a new interconnection technology. IEEE Trans. Parallel Distrib. Syst., 4:3-12, 1993.

[4] A. Ilić, S. Klavžar and Y. Rho. Generalized Fibonacci cubes. Discrete Math., 312:2$11,2012$.

[5] A. Ilić, S. Klavžar and Y. Rho. The index of a binary word. Theoret. Comput. Sci., 452:100-106, 2012.

[6] S. Klavžar. Structure of Fibonacci cubes: a survey. to appear in J. Comb. Optim., doi:10.1007/s10878-011-9433-z.

[7] S. Klavžar and S. Shpectorov. Asymptotic number of isometric generalized Fibonacci cubes. European J. Combin., 33:220-226, 2012.

[8] J. Liu, W.-J. Hsu and M. J. Chung. Generalized Fibonacci cubes are mostly Hamiltonian. J. Graph Theory, 18:817-829, 1994.

[9] D. Merlini and R. Sprugnoli. Algebraic aspects of some Riordan arrays related to binary words avoiding a pattern. Theoret. Comput. Sci., 412:2981-3001, 2011.

[10] R. Sedgewick and P. Flajolet. An Introduction to the Analysis of Algorithms. Addison-Wesley, Reading, MA, 1996.

[11] N. J. A. Sloane. The On-Line Encyclopedia of Integer Sequences. Published electronically at http://oeis.org.

[12] N. Zagaglia Salvi. On the existence of cycles of every even length on generalized Fibonacci cubes. Matematiche (Catania), 51:241-251, 1996. 